\title{
CORRECTION
}

\section{Correction to: Efficacy and safety of stress ulcer prophylaxis in critically ill patients: a network meta-analysis of randomized trials}

\author{
Waleed Alhazzani ${ }^{1,2 *}$, Fayez Alshamsi ${ }^{3}$, Emilie Belley-Cote ${ }^{2}$, Diane Heels-Ansdell ${ }^{2}$,

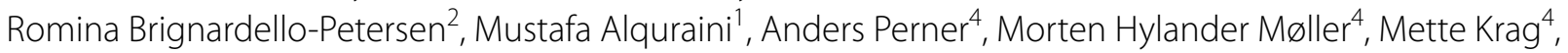 \\ Saleh Almenawer ${ }^{5}$, Bram Rochwerg ${ }^{1,2}$, Joanna Dionne ${ }^{1}$, Roman Jaeschke ${ }^{1,2}$, Mohammed Alshahrani ${ }^{6}$, \\ Adam Deane ${ }^{7}$, Dan Perri ${ }^{1}$, Lehana Thebane ${ }^{2}$, Awad Al-Omari ${ }^{8,9}$, Simon Finfer ${ }^{10}$, Deborah Cook ${ }^{1,2}$ \\ and Gordon Guyatt ${ }^{1,2}$
}

(c) 2017 Springer-Verlag GmbH Germany, part of Springer Nature and ESICM

\section{Correction to: Intensive Care Med} https://doi.org/10.1007/s00134-017-5005-8

Owing to an oversight by the authors, all the figures in the last column of Table 2 (upper section: Number needed to treat; lower section: Number needed to harm) in this article were given without the final digit 0 and are thus 10 times too small.

The corrected Table 2 is shown here.

The authors regret any inconvenience caused.

*Correspondence: alhazzaw@mcmaster.ca

${ }^{1}$ Division of Critical Care, Department of Medicine, McMaster University,

St Joseph's Healthcare Hamilton, 50 Charlton Avenue, Hamilton, ON L8N

4A6, Canada

Full author information is available at the end of the article

The original article can be found online at https://doi.org/10.1007/

s00134-017-5005-8.

\section{第 Springer}


Table 2 Absolute treatment effect for clinically important bleeding and pneumonia outcomes

\begin{tabular}{|c|c|c|}
\hline \multicolumn{3}{|c|}{ Clinically important GI bleeding } \\
\hline Comparison & RD per 1000 patients $\left(95 \%\right.$ CI) for ACR 2.1 for placebo $\%^{a}$ & Number needed to treat \\
\hline H2RA vs placebo & 8 fewer per 1000 (6 more to 14 fewer) & 130 \\
\hline PPI vs H2RA & 8 fewer per 1000 (from 4 fewer to 10 fewer) & 130 \\
\hline H2RA vs sucralfate & 3 fewer per 1000 (from 7 more to 9 fewer) & 330 \\
\hline PPI vs placebo & 16 fewer per 1000 (from 8 fewer to 19 fewer) & 60 \\
\hline Sucralfate vs placebo & 4 fewer per 1000 (from 13 fewer to 15 more) & 250 \\
\hline PPI vs sucralfate & 12 fewer per 1000 (from 6 fewer to 15 fewer) & 80 \\
\hline \multicolumn{3}{|l|}{ Pneumonia outcome } \\
\hline Comparison & RD per 1000 patients $(95 \% \mathrm{Cl})$ for $\mathrm{ACR} 6 \%$ in placebo ${ }^{\mathrm{b}}$ & Number needed to harm \\
\hline H2RA vs placebo & 11 more per 1000 (from 12 fewer to 42 more) & 90 \\
\hline PPI vs H2RA & 19 more per 1000 (from 3 fewer to 48 more) & 50 \\
\hline H2RA vs sucralfate & 17 more per 1000 (from 4 more to 32 more) & 50 \\
\hline PPI vs placebo & 31 more per 1000 (from 3 fewer to 85 more) & 30 \\
\hline Placebo vs sucralfate & 5 more per 1000 (from 15 fewer to 36 more) & 200 \\
\hline PPI vs sucralfate & 36 more per 1000 (from 11 more to 70 more) & 30 \\
\hline
\end{tabular}

$R D$ risk difference, $A C R$ assumed control event rate, $H 2 R A$ histamine-2 receptor antagonists, $P P I$ proton pump inhibitor, $G I$ gastrointestinal

a The median event rate of clinically important bleeding across all trials in placebo arm was $2.1 \%$

b The median event rate of pneumonia across all trials in placebo arm was $6 \%$

\section{Author details}

${ }^{1}$ Division of Critical Care, Department of Medicine, McMaster University, St Joseph's Healthcare Hamilton, 50 Charlton Avenue, Hamilton, ON L8N 4A6, Canada. ${ }^{2}$ Department of Health Research Methods, Evidence, and Impact, McMaster University, Hamilton, Canada. ${ }^{3}$ Department of Internal Medicine, College of Medicine and Health Sciences, UAE University, Al Ain, United Arab Emirates. ${ }^{4}$ Department of Intensive Care, Copenhagen University Hospital Rigshospitalet, Copenhagen, Denmark. ${ }^{5}$ Department of Surgery, McMaster University, Hamilton, Canada. ${ }^{6}$ Department of Critical Care, Dammam University,
Dammam, Saudi Arabia. ${ }^{7}$ Discipline of Acute Care Medicine, University of Adelaide, Adelaide, Australia. ${ }^{8}$ Department of Critical Care, Security Forces Hospital, Riyadh, Saudi Arabia. ${ }^{9}$ Department of Medicine, Alfaisal University, Riyadh, Saudi Arabia. ${ }^{10}$ The George Institute for Global Health and Royal North Shore Hospital, University of Sydney, Sydney, NSW, Australia.

Published online: 11 December 2017 